\title{
Supernova Optical Observations and Theory
}

\author{
Keiichi Maeda, Melina C. Bersten, Takashi J. Moriya, \\ Gaston Folatelli, and Ken'ichi Nomoto
}

Kavli Institute for the Physics and Mathematics of the Universe, University of Tokyo, 5-1-5 Kashiwanoha, Kashiwa, Chiba 277-8583, Japan

email: keiichi.maeda@ipmu.jp

\begin{abstract}
We review emission processes within the supernova (SN) ejecta. Examples of the application of the theory to observational data are presented. The emission processes and thermal condition within the SN ejecta change as a function of time, and multi-epoch observations are important to obtain comprehensive views. Through the analyses, we can constrain the progenitor radius, compositions as a function of depth, ejecta properties, explosion asymmetry and so on. Multi-frequency follow-up is also important, including radio synchrotron emissions and the inverse Compton effect, $\gamma$-ray emissions from radioactive decay of newly synthesized materials. The optical data are essential to make the best use of the multi-frequency data.
\end{abstract}

Keywords. supernovae: general, radiation mechanisms: general.

\section{Introduction}

Supernovae (SNe) are classified into several types by characteristics in their spectra around the maximum light (e.g., Filippenko 1997). The spectral typing has been linked to the progenitor scenarios through the amount of the envelope present at the time of explosion (e.g., Nomoto et al. 1995). Stars that retain their $\mathrm{H}$ envelope produce SNe with H-rich spectra, classified as SNe II. SNe II are divided into a few sub categories. If there are strong hydrogen (narrow) emission lines, an SN is classified as SN IIn. Stars that have lost at least a large fraction of the $\mathrm{H}$ envelope produce He-rich $\mathrm{SNe}$ IIb, Hdeficient $\mathrm{SNe} \mathrm{Ib}$, and both $\mathrm{H}$ and He-deficient SNe Ic, in order of increasing degree of the envelope stripping (called stripped-envelope SNe; SE-SNe). They are believed to be a core-collapse (CC) SNe from a massive star. SNe Ia show neither H nor He, and they are characterized by a strong $\mathrm{Si}$ absorption. This matches well the standard scenario that $\mathrm{SNe}$ Ia are a result of a thermonuclear explosion of a $\mathrm{C}+\mathrm{O}$ white dwarf, reaching (nearly) the Chandrasekhar limiting mass (e.g., Nomoto et al. 1984; Woosley \& Weaver 1986).

\section{Optical Emission Processes and Model Examples}

Generally, an SN is described as a metal-rich fireball, homologously expanding into a space with $\sim 10,000 \mathrm{~km} \mathrm{~s}^{-1}$. Thus the optical depth decreases with time. Photosphere moves back toward the center as time goes by, and so does the line forming region until the photosphere disappears. This property allows one to extract the chemical and structure information within the ejecta as a function of the depth (Stehle et al. 2006).

$\mathrm{SNe}$ of different types are also associated with different energy sources. In the beginning, the energy source could be the thermal energy deposited at the explosion. The thermal energy is more important for SNe with a more extended envelope. This is a dominant source in SNe IIp for the first $\sim 100$ days, while it lasts only for a few days in SE SNe. SNe create a huge amount of radioactive ${ }^{56} \mathrm{Ni}$, and its decay is another major 

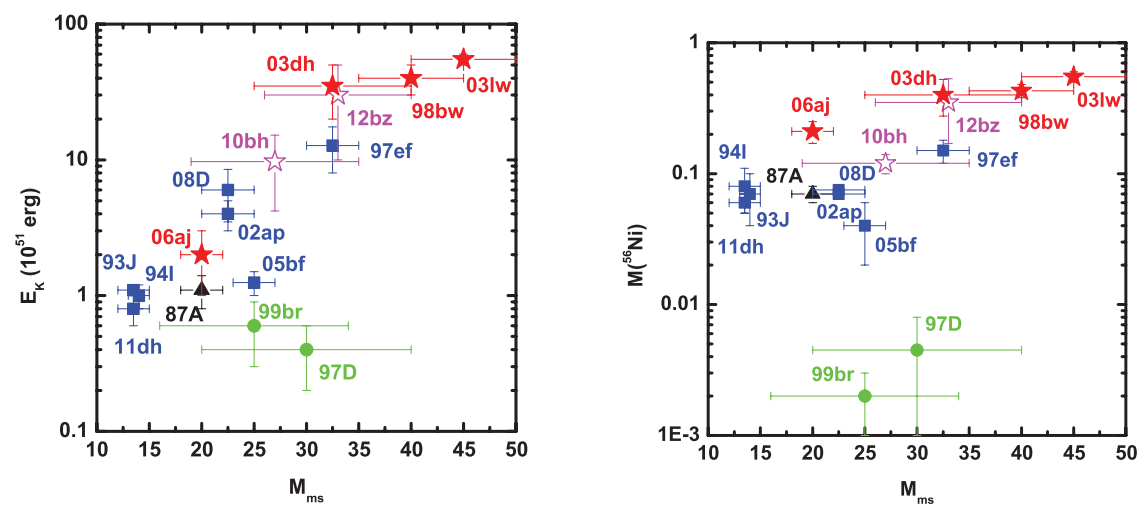

Figure 1. Relations in $M_{\mathrm{ms}}-E_{\mathrm{K}}$ and $M_{\mathrm{ms}}-M\left({ }^{56} \mathrm{Ni}\right)$. Filled symbols are for those derived with numerical radiation transfer modeling, while open symbols are those estimated with simple scaling relations. Shown here are GRB-SNe Ic (red/magenta stars), SE SNe (blue squares), faint SNe IIP (green circles), and SN 1987A (black triangle).

source. If the CSM density is high, the energy input from the ejecta-CSM interaction could dominate, and this is believed to be a power source of SNe IIn.

\subsection{Maximum-Light Analysis}

Details in analyzing the observed properties around the maximum brightness are different for different types of SNe, and here we show a specific example of SE SNe. In these ${ }^{56} \mathrm{Ni}$ powered SNe with little or no H-envelope, the peak luminosity $(L)$, light curve time scale $(\Delta t)$, and the expansion velocity from absorption features $(V)$, are connected to the mass of ${ }^{56} \mathrm{Ni}\left[M\left({ }^{56} \mathrm{Ni}\right)\right]$, ejecta mass $\left(M_{\mathrm{ej}}\right)$, the kinetic energy of the explosion $\left(E_{\mathrm{K}}\right)$, as follows: $L \propto M\left({ }^{56} \mathrm{Ni}\right), \Delta t \propto E^{-1 / 4} M^{3 / 4}, V \propto E_{\mathrm{K}}^{1 / 2} M_{\mathrm{ej}}^{-1 / 2}$. The main-sequence mass $\left(M_{\mathrm{ms}}\right)$ can be estimated from $M_{\mathrm{ej}}$. Results from this kind of analyses are shown in Figure 1.

There is a trend that a more massive progenitor results in a more energetic explosion with a large amount of ${ }^{56} \mathrm{Ni}$. Some SNe Ic show broad absorption features, indicating a larger energy than other $\mathrm{SNe}\left(E_{\mathrm{K}} \sim 10^{51} \mathrm{erg}\right)$ (e.g., Iwamoto et al. 1998). The most energetic broad-line SNe Ic reach $E_{\mathrm{K}} \gtrsim 10^{52} \mathrm{erg}$, and some of them are associated with long-soft Gamma-Ray Bursts (hereafter GRBs) (Galama et al. 1998; Hjorth et al. 2003).

\subsection{Early-Phase Emission}

There are an increasing number of SNe discovered just after the explosion (e.g., Nugent et al. 2011). Such an early emission is sensitive to the outer envelope structure of the progenitor. An SN from a more extended progenitor is predicted to show a higher luminosity persisting for a longer time scale. A specific example here is shown for SN IIb 2011dh in M51. The SN properties around maximum are very similar to a prototypical SN IIb 1993J. Figure 2 (left) shows an example of the maximum light modeling (§2.1). The He core mass was derived to be $\sim 4 M_{\odot}$, the explosion energy to be $\sim 0.8 \times 10^{51}$ erg, and $M\left({ }^{56} \mathrm{Ni}\right) \sim 0 . .06 M_{\odot}$ (Bersten et al. 2012). Thus SN 2011dh is almost a twin of SN1993J. The main sequence mass must have been $\sim 12-15 M_{\odot}$. This suggests that the binary evolution has to be invoked to have the hydrogen envelope stripped away.

Pre-explosion HST images show that unexpectedly there was an yellow supergiant (YSG) at the SN position (Maund et al. 2011; Van Dyk et al. 2011). While there had been discussion about whether this was a progenitor or not, later images showed that the YSG has disappeared-it was a progenitor (Van Dyk et al. 2013). Before this confirmation, 

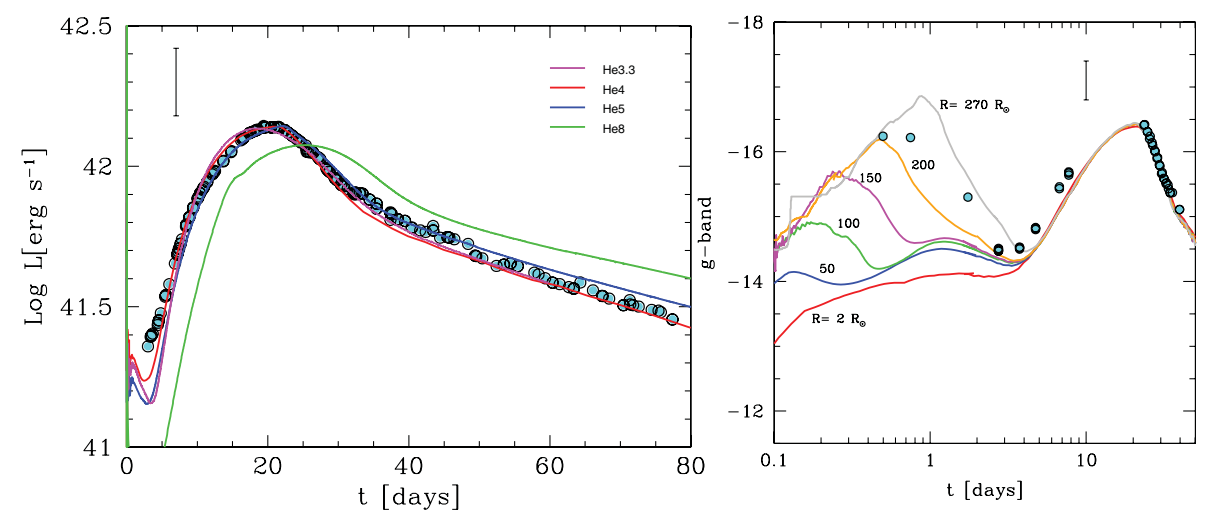

Figure 2. Radiation hydrodynamic simulations for SN 2011dh (Bersten et al. 2012). Left panel shows the maximum-light analysis. Right panel shows the early-phase analysis.

Bersten et al. (2012) analyzed the early emission to address the issue. Figure 2 (right) shows the early-phase light curve compared to the models with different progenitor radii. There is the initial emission from the shock-deposited thermal energy clearly detected, which requires the progenitor as large as $\sim 200 R_{\odot}$, consistent with the YSG.

The very early phase spectra also contain the abundance information at the progenitor surface. Recent highlights include a discovery of carbon lines in many SNe Ia (Parrent et al. 2011; Folatelli et al. 2012). The carbon content is a key in clarifying progenitor and explosion scenarios (Maeda et al. 2010b; Tanaka et al. 2011).

Another example is about Super Luminous SNe (SLSNe). While the leading model for its power is an SN-CSM interaction within a very dense CSM (Moriya et al. 2013), a few other scenarios such as a magnetor input model have not been rejected (Kasen et al. 2010; see also Maeda et al. 2007a). A new approach to discriminate the interaction model from the others has been proposed by Moriya \& Maeda (2012), which predicts a brief 'dip' before the LC rise as was indicated for SNSN 2006oz (Leloudas et al. 2012).

\subsection{Late-Phase Emission}

Late-time observations also have unique advantage. The SN ejecta become fully transparent in about a year. At this phase the emission from the innermost region is directly viewed by an observer. It thus provides a unique way to probe the abundance and structure of the innermost region, which are closely related to the nature of the explosion. Deriving the explosion geometry through a late-time spectrum is a unique and powerful way to tackle to the explosion physics, and this will be separately discussed in $\S 3$.

As an example, Figure 3 shows a late-time spectrum of SN 2006aj (Maeda et al. 2007b), which was discovered in association with an X-Ray Flash (XRF) 060218 (Pian et al. 2006). Unlike typical SE SNe, the feature at $7400 \AA$ is identified as $[\mathrm{Ni} \mathrm{II}]$, not [Ca II]. It was then derived that the amount of stable ${ }^{58} \mathrm{Ni}$ is $\sim 0.05 M_{\odot}$. This large amount of neutron-rich materials may indicate that there was a strong wind from a newly-formed neutron star, and such a strong activity might well be linked to a birth of the XRF.

SN Ia explosion physics is another application. Maeda et al. (2010a) examined latetime spectra of SNe Ia and concluded that one can associate different thermonuclear burning products to different emission lines: [Fe III] blend at $\sim 4700 \AA$ comes from a low-density ${ }^{56}$ Ni-rich region, while [Fe II] $\lambda 7155$ and [Ni II] $\lambda 7388$ are from a high-density, stabile $\mathrm{Fe} / \mathrm{Ni}$-rich region. The existence of stable Fe-peaks could be a smoking gun to distinguish several popular progenitor and explosion models (Maeda et al. 2010b). 


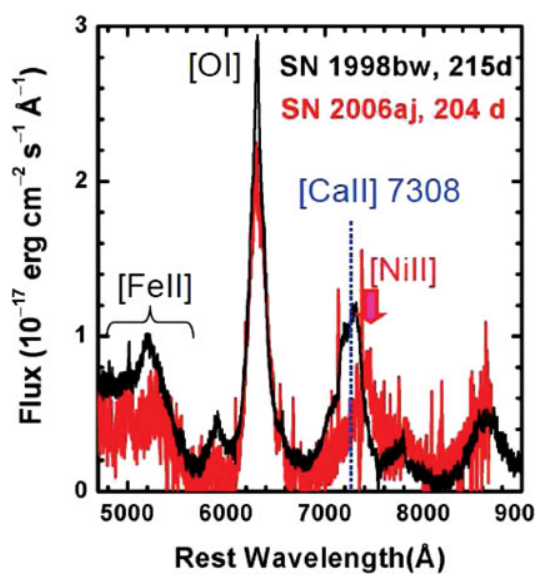

Figure 3. A late-time spectrum of SN 2006aj (Maeda et al. 2007b).

Late-time observations have led to new discoveries as well. SN Ib 2005cz was peculiar, as this was discovered in an elliptical galaxy. Similar objects are also found in elliptical or outskirts. Except for being faint $\left[M\left({ }^{56} \mathrm{Ni}\right) \sim 0.01 M_{\odot}\right]$, the early-phase spectral evolution was not peculiar as SE SNe. However, their late-time spectra are characterized by a lack of [OI] and by strong [Ca II] (Kawabata et al. 2010). The origin of these 'Ca-rich' SNe is still under debate (Parets et al. 2010), including suggestion that it is an explosion of a star at the lower boundary to become an $\mathrm{SN}\left(M_{\mathrm{ms}} \sim 8-10 M_{\odot}\right)$ (Kawabata et al. 2010$)$.

\section{Explosion asymmetry}

Explosion mechanisms of SNe have not been clarified. The geometry is probably a key. Different scenarios predict different types of explosion geometry, thus observationally deriving the geometry of the innermost ejecta is of highest importance. The ejecta become transparent at about 100 or 200 days after the explosion. Thanks to the homologous expansion, the Doppler shift indicates where the photon was emitted. A photon emitted from the near/far side of the ejecta is blueshifted/redshifted. Thus, the line profile can be used as a direct tracer of the distribution of the emitting materials.

In SE SNe, [O I] $\lambda \lambda 6300,6363$ is the strongest in their late-time spectra. Maeda et al. (2002) predicted that a bipolar explosion should produce double peaks in the [O I] if viewed from the equatorial direction. Figure 4 (left) shows the [O I] line profiles of 18 SE SNe we have obtained by Subaru and VLT (Maeda et al. 2008). We found that the doubly-peaked profile is not rare, suggesting that the asphericity is a generic feature in SE SNe, and a bipolar-type geometry is a straightforward interpretation.

We have also investigated explosion asymmetry in SNe Ia through late-time spectra, based on the idea that the distribution of different burning products can be traced by line profiles of different lines (§2.3). We discovered that $[\mathrm{Fe}$ II $] \lambda 7155$ and $[\mathrm{Ni}$ II] $] \lambda 7378$ do show variations in its central wavelength for different SNe Ia; some SNe show redshift and others blueshift. The above observations indicate that an asymmetric, offset ignition is a generic feature of SNe Ia (Maeda et al. 2010a; Maeda et al. 2011).

The geometry derived here could be a key to understanding various diverse properties of SNe. For example, it has been pointed out that normal SNe can be categorized into two groups based on the Si absorption feature, i.e., the high velocity group and low velocity group (Benetti et al. 2005). In addition, SNe Ia show a variation in their peak colors 

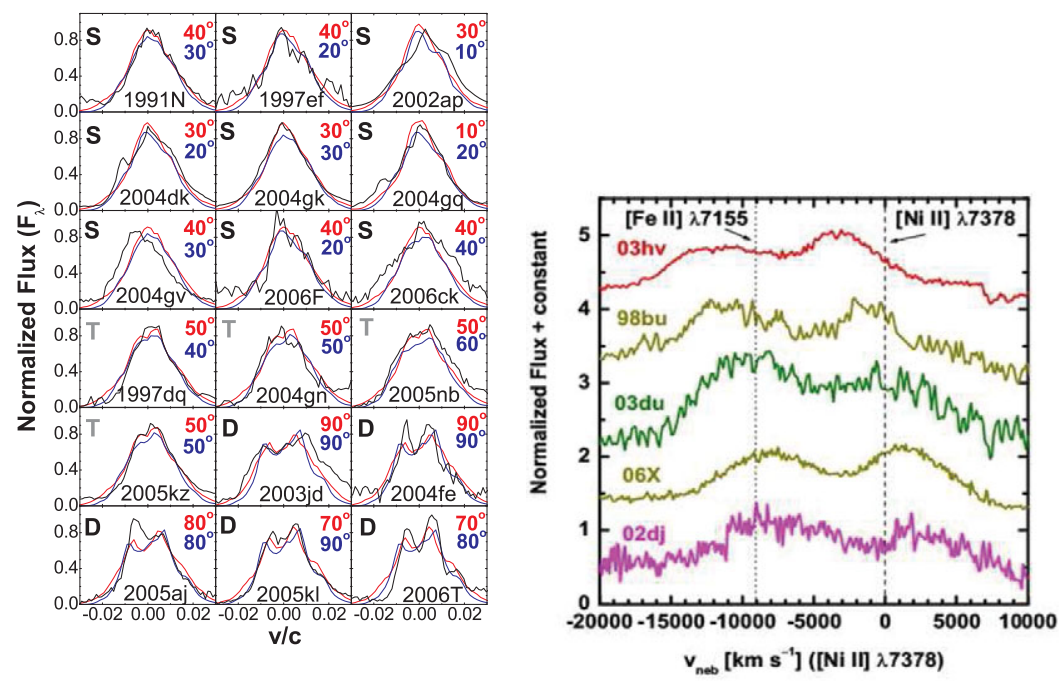

Figure 4. Left: line profiles of [OI] in late-time spectra of SE SNe (black) (Maeda et al. 2008). Also shown are the bipolar explosion model predictions (Maeda et al. 2002; Maeda et al. 2006). Right: Late-time spectra of some selected SNe Ia (Maeda et al. 2010a; Maeda et al. 2011). .

(e.g., Folatelli et al. 2010). The origin of these diversities has not yet been clarified, and solving these could have a high impact in the SN cosmology.

We discovered that the Si velocity in the early phase is correlated to the velocity shift in the nebular lines in the late-phases (Figure 5: Maeda et al. 2010c). The correlation indicates that there is no intrinsic difference between high velocity $\mathrm{SNe}$ and (at least a part of) low velocity $\mathrm{SNe}$, but the different appearance is a consequence of different viewing directions. At the same time, there has been accumulating evidence that a part of low-velocity SNe belong to a population intrinsically different than high velocity SNe (Wang et al. 2013). Combining all these results suggests that there are likely (at least) two populations in normal SNe Ia, one including both high velocity and low velocity $\mathrm{SNe}$, while the other including only low velocity SNe Ia. The late-time spectroscopic study suggests that there is not intrinsic difference in the high velocity and low velocity $\mathrm{SNe}$ in the former group, and may well be explained by the viewing angle effect.

SNe Ia show a variation in their peak colors. This is another major issue in SN cosmology since the estimate of extinction (thus distance) relies on the intrinsic color. By comparing the 'viewing direction' and the color, we have shown that that the intrinsic color variation is at the level of $B-V \sim 0.2 \mathrm{mag}$, and the variation within this level can be attributed to the intrinsic variation (Maeda et al. 2011; Cartier et al. 2011).

\section{Optical Study in Multi-wavelength Context}

Looking at the same object in different wavelengths provides a comprehensive view of astrophysical objects, and it is also the case in SNe. Radio synchrotron emissions from SNe (so far detection limited for CC SNe) provide not only the CSM density/distribution but also the ejecta mass and the kinetic energy (Maeda 2012a; Maeda 2013a). Figure 6 shows a relation between the peak date and peak luminosity at $5 \mathrm{GHz}$. SNe with the same set of the ejecta mass and the kinetic energy should follow a line in this diagram. So far the observational data indicate that SE SNe have quite uniform ejecta properties, 


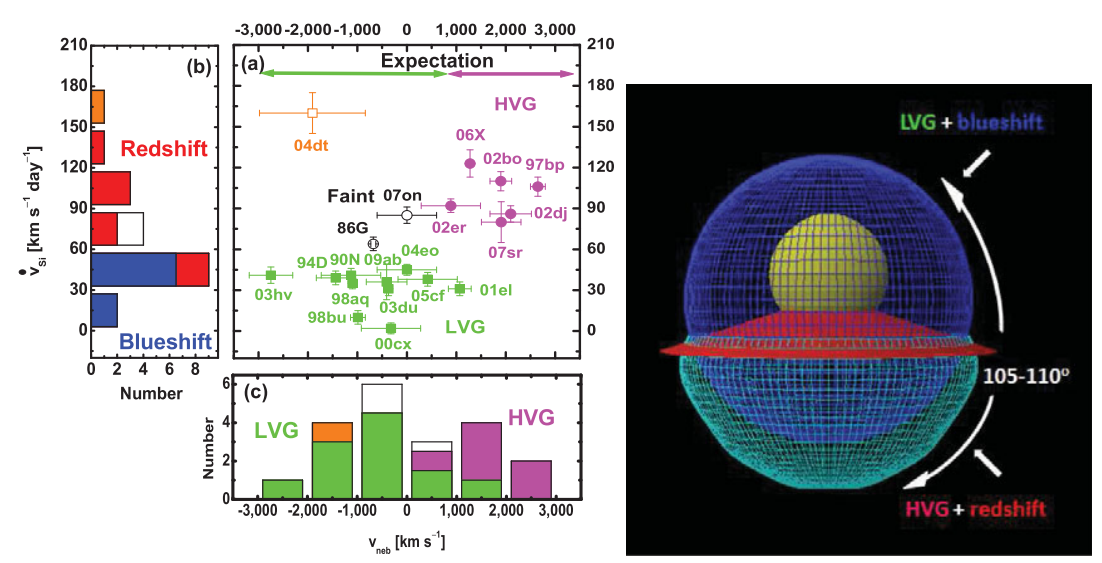

Figure 5. The left panel shows a comparison between the velocity gradient $\left(\dot{v}_{\mathrm{Si}}\right)$ and the latetime emission line velocity shift $\left(v_{\mathrm{neb}}\right)$ (Maeda et al. 2010c). The right panel shows a schematic picture of SN Ia ejecta derived from the late-time spectra and its relation to the velocity.

with the ejecta mass seemingly clustered into $\sim 1-5 M_{\odot}$. This may indicate that the binary path could be important. This can be compared to the insight from the optical data provided through the maximum light analysis (§2.1). For example, in case of SN IIb 2011dh, the radio multi-band light curves can be explained quite well by adopting the ejecta properties obtained through the optical analysis (Maeda 2012a).

Combining the optical data with other wavelengths is critical in understanding the shock wave physics. The optical data directly provide the thermal photon density at the shock wave. Depending on the density of relativistic electrons there, one expects to see the inverse Compton cooling effect in the radio emission. By this way, one can constrain the relativistic electron population at the shock wave, as suggested by Maeda (2013a) (see also Maeda 2012a). Analyzing a few SNe, the efficiency of the electron acceleration is found to be less than $1 \%$ (i.e., less than $1 \%$ of the shock heated thermal energy goes into the relativistic electrons). This is an important input in constraining the electron energy distribution at the SN shock through multi-wavelength study (Maeda 2013b).

Another example is the high energy emission from radioactive decay. Nearby SNe Ia are within reach by NuStar and Astro-H in hard X and soft $\gamma$-rays (Fig. 7: Maeda et al. $2012 \mathrm{~b}$ ), hopefully leading to detection of radioactive decay chain ${ }^{56} \mathrm{Ni} \rightarrow{ }^{56} \mathrm{Co} \rightarrow{ }^{56} \mathrm{Fe}$ in the near future. This will be direct evidence of the syntheses of ${ }^{56} \mathrm{Ni}$ and its amount. This will provide a test for our analysis of optical data (e.g., §2.1) through a totally independent way, and this could bring us a better understanding of the nature of SNe.

\section{Summary}

We have reviewed emission processes within SN ejecta. Various examples of applying the theory to observational data have been presented. The emission processes and thermal condition of the SN ejecta change as a function of time, and thus multi-epoch follow-up is important to obtain comprehensive views. The very early phase observations can be used to estimate the progenitor radius and surface compositions, both are closely related to the evolution toward an SN. Such an early follow-up may also shed light on still unresolved nature of SLSNe. Maximum-light properties have been leading to relations between various explosion/progenitor parameters. Late-phase observations are critical to 


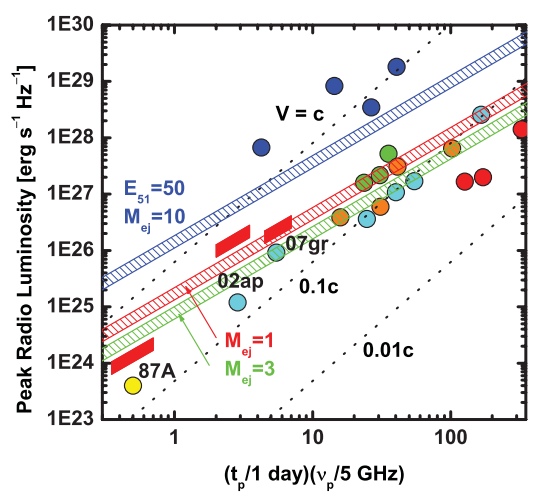

Figure 6. Radio properties of SE SNe. The peak luminosity is plotted as a function of the peak date. See Maeda (2013a) for details. The expected constant lines for a given set of ejecta properties are shown by the shaded area (blue, red, and green, with $E_{51}$ and $M_{\mathrm{ej}}$ given in labels).
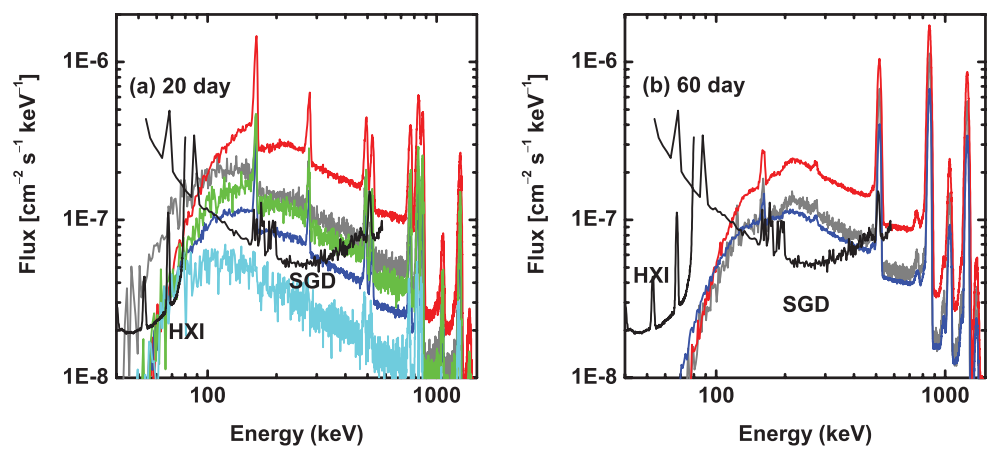

Figure 7. Exampels of synthetic high-energy spectra at (a) 20 days and (b) 60 days after the SN Ia explosion (at $10 \mathrm{Mpc}$ ). Sensitivity curves $\left(10^{6}\right.$ seconds) of $H X I$ and $S G D$ on board Astro-H are shown by black lines. See Maeda et al. $2012 \mathrm{~b}$ for details.

tackle to the still unresolved nature of SN explosions, as the late-phase emission is a probe to materials deep in the ejecta, which directly reflect the nature of the explosion,

Application of the late-time data is highlighted by deriving the geometry of the explosion, We have shown that both CC SNe and SNe Ia are asymmetric, but a way how it deviates from spherically symmetry is different. This interpretation offers the asymmetry and viewing direction as a possible origin of some observational diversities of SNe.

Not only multi-epoch, but also multi-frequency follow-up is important. Ejecta properties as estimated from the optical data analysis can be tested with radio data in an independent way. Further, considering the cooling effect in radio has been suggested to place a strong constraint on the efficiency of relativistic electron acceleration at the SN shock wave, and the analysis requires the optical data as an input. In the near future, we also expect to detect and analyze hard X and soft $\gamma$-rays from radioactive isotopes produced at the SN explosions, and a combined analysis of such data and the optical data will lead to a comprehensive understanding of the SN progenitors and explosions. 


\section{Acknowledgements}

This research is supported by World Premier International Research Center Initiative (WPI Initiative), MEXT, Japan and by Grant-in-Aid for Scientific Research (23740141).

\section{References}

Benetti, S., et al. 2005, ApJ, 623, 1011

Bersten, M. C., et al. 2012, ApJ, 757, 31

Cartier, R., et al. 2011, A\&A A, 534, L15

Filippenko, A. V. 1997, ARAA, 35, 309

Folatelli, G., et al. 2010, $A J, 139,120$

Folatelli, G., et al. 2012, ApJ,745, 74

Galama, T. J., et al. 1998, Nature, 395, 670

Hjorth, J., et al. 2003, Nature, 423, 847

Iwamoto, K., et al. 1998, Nature, 395, 672

Kasen, D. \& Bildsten, I. 2010, ApJ, 717, 245

Kawabata, K. S., et al. 2010, Nature, 465, 326

Leloudas, G., et al. 2012, A\&A A, 541, L129

Maeda, K., et al. 2002, ApJ, 565, 405

Maeda, K., et al. 2006, ApJ, 640, 854

Maeda, K., et al. 2007a, ApJ, 666, 1069

Maeda, K., et al. 2007b, ApJ, 658, L5

Maeda, K., et al. 2008, Science, 319, 1220

Maeda, K., et al. 2010a, ApJ, 708, 1703

Maeda, K., et al. 2010b, ApJ, 712, 624

Maeda, K., et al. 2010c, Nature, 466, 82

Maeda, K., et al. 2011, MNRAS, 413, 3075

Maeda, K. 2012a, ApJ, 758, 81

Maeda, K., et al. 2012b, ApJ, 760, 54

Maeda, K. 2013a, ApJ, 762, 14

Maeda, K. 2013b, ApJ, 762, L24

Maund, J. R., et al. 2011, ApJ, 739, L37

Moriya, T. J. \& Maeda, K. 2012, ApJ, 756, L22

Moriya, T. J., et al. 2013, MNRAS, 428, 1020

Nomoto, K., Thielemann, F.-K., \& Yokoi, K. 1984, ApJ, 286, 644

Nomoto, K., Iwamoto, K., \& Suzuki, T. 1995, Phys. Rep., 256, 173

Nugent. P., et al. 2011, Nature, 480, 344

Parets. H. B., et al. 2010, Nature, 465, 322

Parrent. J. T., et al. 2011, ApJ, 732, 30

Pian, E., et al. 2006, Nature, 442, 1011

Stehle, M., et al. 2006, MNRAS, 360, 1231

Tanaka, M., et al. 2011, MNRAS, 410, 1725

Van Dyk, S. D., et al. 2011, ApJ, 741, L28

Van Dyk, S. D., et al. 2013, ATEL, 4850

Wang, X., et al. 2013, Science, 340, 170

Woosley, S. E. \& Weaver, T. A. 1986, ARAA, 24, 205

\section{Discussion}

TURATTO: Is it correct that the best spectral range for investigating the assymmetries in SNR is the NIR? 
MAEDA: Yes, the NIR can be a better place to see than the optical. The line blend is still there, but solving the blend is easier is the NIR than in the optical. Observationally the NIR is tough, but we are running NIR late-time observations to increase the late-time NIR spectra. 